\title{
Dialektische Methodologie vor dem Hintergrund planetarischer Urbanisierung
}

Rezension zu Neil Brenner (2019): New Urban Spaces. Urban Theory and the Scale

Question. New York: Oxford University Press

Rabea Berfelde

Abb. 1 Titel des Buches (Oxford University Press)

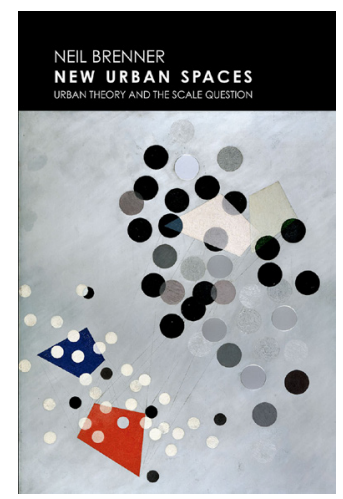

Neil Brenners New Urban Spaces ist eine anspruchsvolle Lektüre mit einem hohen Abstraktionsniveau. Zielgruppe seiner Monographie ist demnach vor allem ein Fachpublikum, welches Interesse an einer theoretisch fundierten Auseinandersetzung mit stadtsoziologischen Fragen hat. Wer sich noch nicht mit Brenners Arbeiten befasst hat, wird in diesem Buch eine dichte Zusammenfassung von über dreißig Jahren raumpolitischen Denkens und kritischer Stadttheorie finden. Das Buch besteht hauptsächlich aus bereits veröffentlichten Artikeln, die allerdings in ihrer Analyse aktualisiert und erweitert werden, um den realen Veränderungen in spätkapitalistischen Urbanisierungsprozessen sowie den Entwicklungen in Brenners eigenem Denken gerecht zu werden. Der Autor führt uns also chronologisch und stets selbstkritisch durch die verschiedenen Phasen seiner akademischen Arbeiten und Überlegungen darüber, inwiefern die, urban question ' als ,scale question formuliert werden kann oder nicht.

Dabei nimmt er selbst eine Aufteilung des Buches in drei analytische Unterteile vor (S. 22). Im ersten Teil, bestehend aus Kapitel 1 bis 3, entwickelt Brenner seinen Analyserahmen, der die weiteren Betrachtungen im Buch strukturiert. Im ersten Kapitel argumentiert er, dass die Urban Studies ihren Analysegegenstand, also die Stadt, anhand einer Reihe impliziter oder expliziter binärer geografischer Unterscheidungen, wie dem Lokalen oder Globalen sowie dem Urbanen oder Ruralen, bestimmen würden. Der Analysegegenstand Stadt würde dabei als eine vorgefundene, territorial begrenzte sozial-räumliche Einheit verstanden, die sich in Abgrenzung zu anderen territorialen Einheiten definiere (S. 1-2). Diese Vorgehensweise bezeichnet er als „,methodological cityism“ (S. 13) und argumentiert demgegenüber, dass sich die Urban Studies vielmehr einem prozessualen Verständnis zuwenden sollten, welches Urbanisierungsprozesse in den Blick nimmt und diese in einer Kombination von skalen- und staatstheoretischen Ansätzen analysiert. In den ersten Kapiteln des Buches schlägt er demnach vor, die „scale question“ (S. 7), also die kontinuierliche Produktion und Verschiebung von geografischen Maßstäben, im Zusammenhang mit staatlich-gelenkter und regulierter Raumproduktion zu verstehen. Der städtische Raum wird 
so „nicht durch die Abgrenzung eines territorialen Gebiets“ sondern als „Kristallisation einer sozial-räumlichen Positionalität innerhalb eines breiteren, multiskalaren Beziehungsrahmens"verstanden (S. 3, Übersetzung R. B.). Territorialität und räumliche Einheit sind somit prozessual und relational definiert. Dieses Argument der Relationalität des geografischen Maßstabs bildet das fundamentale theoretische Gerüst seiner Überlegungen zur territorialen Organisation des Kapitalismus sowie der Verschränkung von Stadt- und Staatsraum, welches er im zweiten Kapitel zunächst in Bezugnahme auf die Schriften David Harveys und Henri Lefebvres weiterentwickelt, um es dann im dritten Kapitel noch einmal im Rückgriff auf seine eigenen Arbeiten aus den 1990er Jahren zu erweitern.

Der zweite Teil, bestehend aus Kapitel vier bis sieben, beschäftigt sich mit empirischen Beispielen der Transformationen von politisch-ökonomischen Geografien und kapitalistischer Urbanisierung. So wendet er den zuvor entwickelten Analyserahmen beispielsweise an, um sich mit dem Diskurs der ,global city' (Kapitel 4), den Geografien der sogenannten ,New Economy (Kapitel 5), dem neuen Regionalismus (Kapitel 6) und dem ,Lokalismus' in der wissenschaftlichen Analyse US-Amerikanischer Stadtpolitik (Kapitel 7) auseinanderzusetzen. Brenner beschäftigt sich in diesem zweiten Teil also neben den konkreten Veränderungen kapitalistischer Urbanisierungsprozesse vor allem mit den Klassikern der kritischen Raumforschung, um seine eigene Argumentation der staatlichen Regulierung von Urbanisierungsprozessen sowie der ,urban question' als ,scale question' zu präzisieren. Diese kontinuierliche Kritik und Erweiterung, nicht nur der Positionen anderer Wissenschaftler*innen, sondern auch seiner eigenen, zeigt bereits hier sehr deutlich sein Verständnis von kritischer Theorieproduktion: Theorie sollte nie zeitlich fixierte Wahrheiten postulieren, sondern sich im Dialog mit den Prozessen der kapitalistischen Umstrukturierung und Reterritorialisierung kontinuierlich weiterentwickeln. So stellt der dritte Teil, welcher seine neueren Arbeiten seit etwa 2010 umfasst, dann auch überraschenderweise eine Kritik des skalaren Denkens dar, das er gerade in den vorangegangenen Kapiteln entwickelt hat. In diesen Kapiteln fordert Brenner nicht nur den ,methodological cityism،, sondern ebenfalls den „scale centrism“ (S. 265) zu überwinden. Zunächst führt er das Konzept des ,polymorphic character ein, um zu argumentieren, dass Skalen nur eine Analysekategorie unter vielen sein können, um die Strukturen urbaner Gefüge zu betrachten. Sie konstituieren und transformieren sich, so Brenner, durch vielfältige sozialräumliche Prozesse die neben Prozessen der Skalierung auch mit Konzepten des ,place-making', der Territorialisierung und der Vernetzung verstanden werden sollten (S. 289).

Das Überwinden des ,scale centrism' ist, wie in Kapitel 9 deutlich wird, auch durch seine Arbeiten zur ,planetary urbanization' motiviert.[1] Das Konzept der planetarischen Urbanisierung ist von Lefebvre übernommen, der Anfang der 1970er Jahre die These von der vollständigen Urbanisierung der Gesellschaft entwickelte (Lefebvre 2003). Neil Brenner postulierte, vor allem in seinen gemeinsamen Arbeiten mit Christian Schmid, dass diese vollständige Urbanisierung empirische Realität geworden sei. Das Städtische stelle demnach heute einen planetarischen Zustand dar, in dem alle politisch-ökonomischen und sozial-ökologischen Beziehungen 
verflochten sind. Das bedeutet, dass selbst Räume jenseits traditionell städtischer Agglomerationen wie Transportnetzwerke, Kommunikationsinfrastrukturen, Ressourcenabbaugebiete und natürliche Räume wie Ozeane, Gebirge, Dschungel, die Atmosphäre und so weiter integrale Bestandteile kapitalistischer Urbanisierungsprozesse sind. Das empirische Aufbrechen der Differenz urban/suburban/ländlich verdeutlicht, dass eine negative Definition des Städtischen in Abgrenzung von eben jenen räumlichen Einheiten des Suburbanen und Ruralen nicht mehr möglich ist, und dass die Methodologien und Methoden städtischer Wissensproduktion überdacht werden müssen, um die aufkommenden Formen der Urbanisierung im 21. Jahrhundert kritisch analysieren zu können. Liest man das Buch von der ersten bis zur letzten Seite, kann man demnach die theoretische Denkbewegung des Autors nachvollziehen, warum und wie seiner Meinung nach, im Zuge der sich historisch verändernden kapitalistischen Raumproduktion und Urbanisierungsprozesse, ein Verständnis der Stadt als gegebene räumliche Analyseeinheit zu überwinden ist. Theorie ist also, wie bereits festgestellt, eine sich fortwährend wandelnde Reflektion des sich ebenfalls ständig verändernden kapitalistischen Akkumulationsregimes sowie dessen räumlichen Ausdrucks.

Daraus ergeben sich zwei Lesarten des Buches. Einerseits kann man durch Brenners Ausführungen die sich seit der Weltwirtschaftskrise der 1970er Jahre und dem Ende des fordistisch-keynesianischen Akkumulationsregime kontinuierlich verändernde kapitalistische Raumproduktion nachvollziehen und bekommt durch seine Ausführungen einen fundierten Einblick sowohl in Mainstream-Theoretisierungen und die wichtigsten kritischen Debatten, die diese Veränderungen begleiteten. Andererseits lässt sich New Urban Spaces auch als Exegese der Dialektik als Methodologie der kritischen Stadtforschung lesen und verstehen.[2] Wie bereits erwähnt, entwickelt Brenner seine Theoretisierung des Städtischen fortwährend weiter und übt dabei im Laufe des Buches zumeist auch explizit Selbstkritik an seiner eigenen Analyse von Urbanisierungsprozessen in vorangegangenen Kapiteln. Die im Buch nachvollzogene Denkbewegung plädiert also für eine bewusste Haltung gegenüber der Art und Weise, wie wir das Städtische theoretisieren und spricht sich dagegen aus, eine einzige ontologische Grundlage zu finden, welche die ,urban question' ein für alle Mal regelt. Seine Argumentation widersetzt sich einer linearen Lesart und die Leser*innen sind dazu angehalten, Ideen und Argumente immer wieder neu zu bewerten, während diese im Laufe des Buches verworfen und/oder weiterentwickelt werden.

Das Buch repräsentiert also selbst eine dialektische Denkbewegung. Zudem finden sich vor allem im ersten Teil des Buches explizite Reflektionen über Dialektik als Methodologie der kritischen Urban Theory. Im Zentrum seines hier entwickelten Analyserahmens steht eine reflexive und ergebnisoffene Dialektik, die Brenner in Lefebvres Werk sowie in Karl Marx Einleitung zu den Grundrissen (1953: 5-31) findet. Die zwei Konzepte Realabstraktionen (,real abstractions') und Reflexivität (,reflexivity') sind dabei für Brenners Verständnis von kritischer (Stadt-)Theorie von besonderer Bedeutung. Konzepte, verstanden als Realabstraktionen, sind in ihrem Entstehungskontext an bestimmte historische Formen räumlicher Praktiken und kapitalistischer Urbanisierung gebunden und demnach Ausdruck 
von und Reflektion der Veränderung kapitalistischer Akkumulationsweise und der damit einhergehenden sozial-räumlichen Veränderungen (Brenner 2019: 41). Reflexivität bedeutet für Brenner, dass Kritik immer in ihrem historisch-spezifischen Kontext verstanden werden sollte. Was wiederum im Umkehrschluss bedeutet, dass sich kritische Theorie fortwährend aktualisieren muss, um auf die sich beständig verändernde Geografien kapitalistischer Vergesellschaftung reagieren zu können.

Was sich im Laufe des Buches herauskristallisiert, ist eine fundierte Intervention, die dazu auffordert, den Untersuchungsgegenstand im Zuge der fortwährenden Veränderung von Urbanisierungsprozessen stetig neu zu bestimmen und dabei über den ,methodological cityism ' hinauszugehen. Was in Brenners dialektischer Konzeption meines Erachtens fehlt, und diese Kritik teile ich mit anderen Rezensent*innen seines Buches, ist ein Einbeziehen der Materialität und Politik des städtischen Alltags (Acuto 2020; Addie 2020). Dass er darauf nicht eingeht, ist verwunderlich, da in Lefebvres historisch-materialisitischer Dialektik, welche die Grundlage von Brenners Ausführungen darstellt, die Perspektive des Alltagslebens eine prominente Rolle einnimmt (Lefebvre 2014). Zudem lässt sich kritisieren, dass in seiner Analyse von Transformationsprozessen kaum handelnde Akteure vorkommen. Auch hier bleibt Brenner hinter Lefebvres ursprünglicher Formulierung der Raumproduktion zurück. Lefebvres dialektisches Verständnis der Raumproduktion denkt die Möglichkeit von Raumaneignungen handelnder Subjekte immer mit (vgl. Lefebvre 2006). Brenners New Urban Spaces und vor allem sein hier dargelegtes Verständnis von Reflexivität und Realabstraktionen gibt kritischen Stadtforscher*innen eine gute methodologische Orientierung an die Hand: einen erkenntnistheoretischen Rahmen und ein Verständnis davon, dass Theorie nie in Stein gemeißelt sein sollte, sondern, um kritisch zu bleiben, die sich wandelnde Materialität sozial-räumlicher Veränderungen stets neu befragen muss. Brenners Dialektik kann dabei aber meines Erachtens nur ein Ausgangspunkt sein. Seine Ansätze sollten weiterentwickelt werden in Richtung einer Methodologie für die kritische Stadtforschung, welche die Perspektive des Alltagslebens, die gelebte Erfahrung von Transformationsprozessen sowie die Handlungsfähigkeit von Subjekten mit in den Blick nimmt.

\section{Endnoten}

[1] Die These von der „planetarischen Urbanisierung“ entwickelte Brenner vor allem in den gemeinsamen Arbeiten mit Christian Schmid, siehe Brenner/Schmid 2015. Die kontroverse These führte zu einer angeregten Debatte in der kritischen Stadtforschung. So wurde zum einen aus eher marxistischer Perspektive kritisiert, dass Brenner und Schmids Vorschlag einer neuen „Epistemologie des Urbanen“ die Materialität des Städtischen weitestgehend außer Acht lässt. Brenner und Schmid würden sich demnach also nicht näher damit auseinandersetzen, wie kapitalistische Vergesellschaftung die konkrete Materialität des Urbanen prägt und Lebensrealitäten in städtischen Umgebungen beeinflusst (vgl . Walker 2015). Zum anderen wurde aus feministischer und postkolonialer Perspektive auf die Bedeutung epistemologischer Pluralität für die kritische Stadtforschung hingewiesen. Diese Stimmen kritisierten, dass Brenner und Schmid in der Debatte um die planetarische Urbanisierung nur einen erkenntnistheoretischen Rahmen vorschlügen, der die Veränderung kapitalistischer Akkumulationsweise als einzigen 
Erklärungs- und Begründungszusammenhang setze. Feministische und postkoloniale Kritiken schlugen als Reaktion vor, nicht ausschließlich kapitalistische Ausbeutung zu betrachten, sondern auch in den Blick zu nehmen, wie globale Verstädterung mit patriarchaler, sexueller und rassifizierter Unterdrückung einhergeht (vgl. Oswin 2018; Buckley/Strauss 2016).

[2] Jean-Paul D. Addies englischsprachige Rezension des Buches in dem Journal Regional Studies schlägt eine ähnliche Lesart vor. Der Autor betont ebenfalls, dass die Lektüre von New Urban Spaces vor allem dann spannend und gewinnbringend ist, wenn man sich beim Lesen darauf konzentriert, wie Brenner dialektisch Theorie produziert (Addie 2020).

\section{Autor_innen}

Rabea Berfelde ist Politik- und Kulturwissenschaftlerin und forscht aktuell im Rahmen ihrer Dissertation zu Veränderungen urbaner Produktions- und Reproduktionsräume im Zuge des Plattformkapitalismus und der zunehmenden Finanzialisierung aller Lebensbereiche. rberfoo1@gold.ac.uk

\section{Literatur}

Acuto, Michele (2020): Book Review. Neil Brenner 2019: New Urban Spaces. Urban Theory and the Scale Question. In: International Journal of Urban and Regional Research 44/1, 173-174.

Addie, Jean-Paul D. (2020): Review. New Urban Spaces. Urban Theory and the Scale Question: By Neil Brenner. In: Regional Studies 54/2, 281-282.

Brenner, Neil (2019): New Urban Spaces. Urban Theory and the Scale Question. New York: Oxford University Press.

Brenner, Neil / Schmid, Christian (2015): Towards a New Epistemology of the Urban? In: City 19/2-3, 151-182.

Buckley, Michelle / Strauss, Kendra (2016): With, against and beyond Lefebvre. Planetary Urbanization and Epistemic Plurality. In: Environment and Planning D 34/3, 617-636.

Lefebvre, Henri (2003): The Urban Revolution. Minneapolis: University of Minnesota Press.

Lefebvre, Henri (2006): Die Produktion des Raums. In: Jörg Dünne / Stephan Günzel (Hg.), Raumtheorie. Grundlagentexte aus Philosophie und Kulturwissenschaft. Frankfurt am Main: Suhrkamp, 330-341.

Lefebvre, Henri (2014): Critique of Everyday Life. London/New York: Verso.

Marx, Karl (1953): Grundrisse der Kritik der politischen Ökonomie. Berlin: Dietz.

Oswin, Natalie (2018): Planetary Urbanization. A View from Outside. In: Environment and Planning D: Society and Space 36/3, 540-546.

Walker, Richard (2015): Building a Better Theory of the Urban. A Response to „Towards a New Epistemology of the Urban?" In: City 19/2-3, 183-191. 